\title{
Contents
}

\section{Tautological Algebras of Moduli Spaces of Curves}

Tautological Algebras of Moduli Spaces of Curves

$\begin{array}{ll}\text { Lecture 1. The tautological ring of } M_{g} & 199\end{array}$

Lecture 2. The tautological rings of $\bar{M}_{g, n}$ and of some natural partial compactifications of $M_{g, n}$

Bibliography 



\section{Tautological Algebras of Moduli Spaces of Curves}

Carel Faber 



\section{Tautological Algebras of Moduli Spaces of Curves}

\section{Carel Faber}

\section{Introduction}

These are the lecture notes for my course at the 2011 Park City Mathematical Institute on moduli spaces of Riemann surfaces. The two lectures here correspond roughly to the first and second half of the course.

The subject of the first lecture is the tautological ring $R^{*}\left(M_{g}\right)$ of $M_{g}$. I recall Mumford's definition of the tautological classes and some of his results from [48]. Then I discuss my conjecture on $R^{*}\left(M_{g}\right)$ from [10] and the results obtained on it. Finally, I survey some recent developments indicating that the relations that suffice to prove the conjecture for $g \leq 23$ may not suffice for larger $g$.

The second lecture concerns mainly the tautological ring of $\bar{M}_{g, n}$. Some natural spaces in between $M_{g, n}$ and $\bar{M}_{g, n}$ are discussed as well. I close with some recent results regarding non-tautological cohomology classes and the cohomology of $\bar{M}_{g, n}$ in low genus.

Department of Mathematics, KTH Royal Institute of Technology, 10044 Stockholm, Sweden E-mail address: faber@kth.se

I thank Benson Farb, Dick Hain, and Eduard Looijenga for the organization of the Institute and Dan Petersen for being the TA for my course. 

LECTURE 1

\section{The tautological ring of $M_{g}$}

Let $g \geq 2$ be an integer and let $M_{g}$ be the moduli space of nonsingular curves of genus $g$. Denote by $A^{*}\left(M_{g}\right)$ the Chow ring of $M_{g}$ with rational coefficients. The tautological ring $R^{*}\left(M_{g}\right)$ is defined as the $\mathbb{Q}$-subalgebra of $A^{*}\left(M_{g}\right)$ generated by the tautological classes (whose definition will be recalled in a moment). The image of $R^{*}\left(M_{g}\right)$ in the rational cohomology ring $H^{*}\left(M_{g}\right)$ will be denoted $R H^{*}\left(M_{g}\right)$. A reader who is unfamiliar with the Chow ring may substitute $R H^{*}\left(M_{g}\right)$ for $R^{*}\left(M_{g}\right)$ and will not lose much as a result. Throughout these notes, however, we will use the algebraic degree, which is half of the cohomological degree. (It should also be noted that it is not known whether the map from $R^{*}\left(M_{g}\right)$ to $R H^{*}\left(M_{g}\right)$ can have a nontrivial kernel.)

Mumford's definition $[48, \S 4]$ of the tautological classes $\kappa_{i} \in R^{i}\left(M_{g}\right)$ in this setting is as follows. Let $C_{g}=M_{g, 1}$ be the moduli space of 1-pointed curves of genus $g$. We view $C_{g}$ as the universal curve. We have the map $\pi: C_{g} \rightarrow M_{g}$ that forgets the marked point. Over $C_{g}$, we have the cotangent line bundle $\mathbb{L}$ at the marked point, which equals the relative dualizing sheaf $\omega_{\pi}$. We denote the first Chern class $c_{1}(\mathbb{L}) \in A^{1}\left(C_{g}\right)$ by $K$. Using the ring structure of $A^{*}\left(C_{g}\right)$, we obtain the powers $K^{i} \in A^{i}\left(C_{g}\right)$ for any $i \geq 0$. The map $\pi: C_{g} \rightarrow M_{g}$ is proper, so that we have push-forward maps $\pi_{*}: A^{i+1}\left(C_{g}\right) \rightarrow A^{i}\left(M_{g}\right)$. The tautological class $\kappa_{i}$ is defined via

$$
\kappa_{i}=\pi_{*}\left(K^{i+1}\right) \in A^{i}\left(M_{g}\right) .
$$

Clearly, $\kappa_{0}=2 g-2$; in the sequel, we will sometimes write $\kappa_{0}$ instead of $2 g-2$ and $\kappa_{-1}$ instead of 0 to write relations in a genus independent form.

The tautological ring $R^{*}\left(M_{g}\right)$ is defined as the $\mathbb{Q}$-subalgebra of the rational Chow ring $A^{*}\left(M_{g}\right)$ generated by the $\kappa_{i}(i \geq 1)$.

A second set of very important classes is formed by the Chern classes of the Hodge bundle. The Hodge bundle $\mathbb{E}=\pi_{*}\left(\omega_{\pi}\right)$ is the vector bundle of rank $g$ over $M_{g}$ whose fiber over the isomorphism class $[C]$ is the space $H^{0}\left(C, \omega_{C}\right)$ of regular differentials on $C$. (In fact, $\mathbb{E}$ extends naturally to a vector bundle over $\bar{M}_{g}$, cf. $[48, \S 4]$.) Put $\lambda_{i}=c_{i}(\mathbb{E}) \in A^{i}\left(M_{g}\right)$.

Using the Grothendieck-Riemann-Roch theorem, Mumford [48, §5] computed the Chern character of the Hodge bundle. The computation is done over the moduli space $\bar{M}_{g}$ of stable curves of genus $g$; restricted to $M_{g}$, the result becomes

$$
\operatorname{ch}(\mathbb{E})=g+\sum_{i=1}^{\infty} \frac{B_{2 i} \kappa_{2 i-1}}{(2 i) !} .
$$

(The Bernoulli numbers $B_{2 i}$ are defined by $t /\left(e^{t}-1\right)=\sum_{i=0}^{\infty} B_{i} t^{i} /(i !)$.) In particular, $c h_{2 i}(\mathbb{E})=0$ for $i \geq 1$ (this is in fact true on $\bar{M}_{g}$ ). The vanishing (on $\bar{M}_{g}$ ) implies that $c h_{j}(\mathbb{E})=0$ for all $j \geq 2 g$ (on $\bar{M}_{g}$, hence on $M_{g}$ as well). Cf. Exc. 1 . 
One also finds the following formula for the lambda classes:

$$
\sum_{i=0}^{\infty} \lambda_{i} t^{i}=\exp \left(\sum_{i=1}^{\infty} \frac{B_{2 i} \kappa_{2 i-1}}{2 i(2 i-1)} t^{2 i-1}\right) .
$$

So the lambda classes are polynomials in the odd kappa classes.

Mumford also found further relations between the lambda and kappa classes using geometric properties of nonsingular curves. Both the nature of the relations and the fact that it is not at all clear how to extend these relations to $\bar{M}_{g}$ are features that they have in common with the relations that we will see later in this lecture.

The idea is to use the fact that on a smooth curve of genus $g \geq 2$ the canonical line bundle is generated by its global sections. This translates as the simple statement that the natural map $\pi^{*} \mathbb{E} \rightarrow \omega_{\pi}$ of locally free sheaves on $C_{g}$ is surjective. The kernel $Q_{g-1}$ of this map is therefore locally free of rank $g-1$, so its Chern classes $c_{j}\left(Q_{g-1}\right)$ vanish in degrees $j \geq g$. In other words, the degree $j$ components of the formal expression

$$
\frac{\pi^{*}\left(1+\lambda_{1}+\lambda_{2}+\cdots+\lambda_{g}\right)}{1+K}
$$

vanish for $j \geq g$. Expanding this as a power series, we obtain on $C_{g}$ the relation

$$
K^{g}-K^{g-1} \pi^{*} \lambda_{1}+K^{g-2} \pi^{*} \lambda_{2}+\cdots+(-1)^{g} \pi^{*} \lambda_{g}=0,
$$

as well as its multiples obtained by multiplying with powers of $K$. Pushing down to $M_{g}$, we find the relations

$$
\kappa_{j-1}-\kappa_{j-2} \lambda_{1}+\kappa_{j-3} \lambda_{2}+\cdots+(-1)^{g} \kappa_{j-1-g} \lambda_{g}=0,
$$

for any $j \geq g$. Combining these relations with the earlier relations expressing the lambda classes as polynomials in the odd kappa classes, Mumford [48, §6] obtained the following result:

Theorem 1.1. $R^{*}\left(M_{g}\right)$ is generated by the first $g-2$ kappa classes $\kappa_{1}, \ldots, \kappa_{g-2}$.

(The growth estimate for the Bernoulli numbers that Mumford uses in the final step of his argument can be replaced by a simple congruence property of these numbers; see Exc. 2.)

We now discuss another method for producing relations between the kappa classes. In fact, the method gives many relations between natural classes on moduli spaces of curves with marked points; pushing down these relations to $M_{g}$, one obtains relations between the kappa classes. It appears to be very difficult to carry out an exhaustive analysis of all the relations produced by the method. As we will see, however, a subset of the relations has been analysed to a substantial extent.

The idea is to study the loci of curves with divisors of a certain degree moving in a complete linear system of at least a given dimension, or more precisely, the loci of such curves together with the divisors, viewed as subvarieties of moduli spaces of pointed curves. In many cases, one can write formulas for the classes of such loci in terms of tautological classes. The loci typically have positive-dimensional fibers for the forgetful map to $M_{g}$ and one cannot push forward the class of such a locus directly to find the class in $M_{g}$ of the locus of curves with such divisors. But by cutting (i.e., intersecting) with various tautological divisors, one obtains loci that upon push-forward to $M_{g}$ give the class in $M_{g}$ with a certain multiplicity, which 
often can be computed. By cutting with different sets of divisors, one obtains different tautological expressions for the class in $M_{g}$; equating them, one finds relations between the kappa classes. In fact, since the goal was to obtain tautological relations, one can just as well push forward the class of a locus with positivedimensional fibers and obtain relations right away.

To set this up, we consider the $d$-fold fiber product of $C_{g}$ over $M_{g}$, which we denote by $C_{g}^{d}$. This is the moduli space of $d$-pointed curves $\left(C ; p_{1}, \ldots, p_{d}\right)$, where $C$ is nonsingular of genus $g$ and the $p_{i}$ are $d$ ordered points of $C$, which may coincide. (We find it convenient to work in the situation where the points are ordered, even though we only study divisors, that is, unordered sums of points. It may very well be worthwhile to work out these relations on the symmetric fiber product, or perhaps on the universal Jacobian.) Write $D$ for the effective divisor $p_{1}+\cdots+p_{d}$ on $C$; by abuse of notation, I will sometimes write $(C ; D)$ for a point of $C_{g}^{d}$.

We consider a natural map of vector bundles on $C_{g}^{d}$. The first bundle is the pull-back from $M_{g}$ of the Hodge bundle. We will still denote this by $\mathbb{E}$ (suppressing the pull-back).

The second bundle is the vector bundle $\mathbb{F}_{d}$ of rank $d$. Its fiber at $(C ; D)$ is the vector space $H^{0}\left(C, \omega_{C} / \omega_{C}(-D)\right)=H^{0}\left(C,\left.\omega_{C}\right|_{D}\right)$. Formally, let $\pi: U \rightarrow C_{g}^{d}$ be the universal curve; $U$ is isomorphic to $C_{g}^{d+1}$ and the map $\pi$ comes with $d$ sections $s_{1}$, $\ldots, s_{d}$. Let $\Sigma \subset U$ be the subscheme that is the union (or sum) of the sections. Then $\mathbb{F}_{d}=\pi_{*}\left(\left.\omega_{\pi}\right|_{\Sigma}\right)$. One can think of $\mathbb{F}_{d}$ as a universal $d$-pointed jet bundle.

Since $\mathbb{E}=\pi_{*}\left(\omega_{\pi}\right)$, we have a natural map

$$
\phi_{d}: \mathbb{E} \rightarrow \mathbb{F}_{d}
$$

of vector bundles over $C_{g}^{d}$. At a point $(C ; D)$, the kernel of $\phi_{d}$ is the vector space $H^{0}\left(C, \omega_{C}(-D)\right)$. By Riemann-Roch,

$$
\operatorname{dim} H^{0}(C, \mathcal{O}(D))-\operatorname{dim} H^{0}\left(C, \omega_{C}(-D)\right)=d-g+1 .
$$

So the rank of $\phi_{d}$ is at most $d-r$ if and only if $\operatorname{dim} H^{0}\left(C, \omega_{C}(-D)\right) \geq g-d+r$ if and only if $\operatorname{dim} H^{0}(C, \mathcal{O}(D)) \geq r+1$.

We conclude that

$$
\left\{\operatorname{rk} \phi_{d} \leq d-r\right\}
$$

considered as a subscheme of $C_{g}^{d}$, equals the locus of $d$-pointed curves $(C ; D)$ for which the complete linear system $|D|$ has dimension at least $r$. The expected codimension of this determinantal locus in $C_{g}^{d}$ equals $(g-d+r)(d-d+r)=r(g-d+r)$, and the expected codimension of the locus in $M_{g}$ of curves with a $g_{d}^{r}$ (a linear system of dimension $r$ and degree $d)$ equals $r(g-d+r)-d+r=(r+1)(g-d+r)-g$ (the opposite of the Brill-Noether number $\rho$ ).

Porteous's formula (cf. $[\mathbf{2}, \mathbf{1 9}]$ ) computes the class of the locus $\left\{\operatorname{rk} \phi_{d} \leq d-r\right\}$ if it is either empty or has the expected codimension. The formula is:

$$
\operatorname{class}\left(\left\{\operatorname{rk} \phi_{d} \leq d-r\right\}\right)=\Delta_{r, g-d+r}\left(c_{t}\left(\mathbb{F}_{d}\right) / c_{t}(\mathbb{E})\right) .
$$

Here, $c_{t}$ stands for the total Chern class, viewed as a polynomial in $t$; the quotient $c_{t}\left(\mathbb{F}_{d}\right) / c_{t}(\mathbb{E})$ is viewed as a formal power series in $t$; and

$$
\Delta_{p, q}\left(\sum_{i=0}^{\infty} c_{i} t^{i}\right)=\left|\begin{array}{cccc}
c_{p} & c_{p+1} & \ldots & c_{p+q-1} \\
c_{p-1} & c_{p} & \ldots & c_{p+q-2} \\
\vdots & \vdots & \ddots & \vdots \\
c_{p-q+1} & c_{p-q+2} & \ldots & c_{p}
\end{array}\right| .
$$


We have the following tautological classes on $C_{g}^{d}$ : the kappa classes $\kappa_{i}$, pulled back from $M_{g}$ (again, it will be more convenient to suppress the pull-backs in the notation), and the divisor classes $K_{i}$ and $D_{i, j}$ : the class $K_{i}$ is the pull-back of $K$ via the projection from $C_{g}^{d}$ onto the $i$ th factor, and $D_{i, j}=D_{j, i}($ for $i \neq j$ ) is the class of the diagonal $p_{i}=p_{j}$. For $k>1$, we also put $\Delta_{k}=D_{1, k}+D_{2, k}+\cdots+D_{k-1, k}$.

We know already that the Chern classes $\lambda_{i}$ of $\mathbb{E}$ are tautological. In addition,

$$
c_{t}(\mathbb{E})^{-1}=c_{t}\left(\mathbb{E}^{\vee}\right)=1-\lambda_{1} t+\lambda_{2} t^{2}+\cdots+(-1)^{g} \lambda_{g} t^{g}
$$

(see Exc. 1), so we can work with polynomials instead of power series in the formula for the class of the degeneracy locus. Fortunately, we also have a relatively simple formula for the total Chern class of $\mathbb{F}_{d}$ in terms of the tautological classes:

$$
c\left(\mathbb{F}_{d}\right)=\left(1+K_{1}\right)\left(1+K_{2}-\Delta_{2}\right)\left(1+K_{3}-\Delta_{3}\right) \cdots\left(1+K_{d}-\Delta_{d}\right)
$$

(see Exc. 3). This means that the classes of the degeneracy loci above are tautological — if the conditions in Porteous's formula are satisfied - and we can produce many relations between the kappa classes on $M_{g}$ by means of the indicated method. Moreover, if a certain degeneracy locus is empty, we find a relation on $C_{g}^{d}$, and if a degeneracy locus has positive-dimensional fibers over $C_{g}^{k}$ for some $k<d$, we find a relation on $C_{g}^{k}$ by pushing forward directly.

So far, we have tacitly assumed that the push-forward of a tautological class by a morphism forgetting a point is tautological. Let us make this explicit:

(1) Every monomial in the classes $K_{i}(1 \leq i \leq d)$ and $D_{i, j}(1 \leq i<j \leq d)$ on $C_{g}^{d}$ can be rewritten as a monomial $M$ pulled back from $C_{g}^{d-1}$ times either a single diagonal $D_{i, d}$ or a power $K_{d}^{k}$ of $K_{d}$ by a repeated application of the following substitution rules:

$$
\left\{\begin{aligned}
D_{i, d} D_{j, d} & \rightarrow D_{i, j} D_{i, d} & & (i<j<d) \\
D_{i, d}^{2} & \rightarrow-K_{i} D_{i, d} & & (i<d) \\
K_{d} D_{i, d} & \rightarrow K_{i} D_{i, d} & & (i<d) .
\end{aligned}\right.
$$

Note that $D_{i, d} D_{j, d}=D_{i, j} D_{i, d}, D_{i, d}^{2}=-K_{i} D_{i, d}$, and $K_{d} D_{i, d}=K_{i} D_{i, d}$; the second equation follows from the self-intersection formula and the other ones are clear.

(2) For $M$ a monomial pulled back from $C_{g}^{d-1}$ :

$$
\left\{\begin{aligned}
\pi_{d, *}\left(M \cdot D_{i, d}\right) & =M \\
\pi_{d, *}\left(M \cdot K_{d}^{k}\right) & =M \cdot \kappa_{k-1} .
\end{aligned}\right.
$$

Here $\pi_{d}: C_{g}^{d} \rightarrow C_{g}^{d-1}$ forgets the $d$ th point. Note that $\pi_{d, *}(M)=0$, as it should be, since $\kappa_{-1}=0$.

As a first example, consider the case of $g_{d}^{1}$ 's, one-dimensional linear systems. The expected codimension in $C_{g}^{d}$ is $g-d+1$ and the expected codimension in $M_{g}$ of the locus of curves with a $g_{d}^{1}$ is $g-2 d+2$. It is known that these are the actual codimensions, if $2 \leq d \leq(g+2) / 2$. The class of the locus in $C_{g}^{d}$ is given by Porteous's formula. The locus has 1-dimensional fibers over its image in $M_{g}$, so by pushing down directly, we obtain a relation between the kappa classes in codimension $g-2 d+1$. Alternatively, we can compute the class of the image in $M_{g}$ in two ways: (a) by cutting with $D_{1,2}$, with multiplicity $(2 g-2+2 d)(d-2)$ !; (b) by cutting with $K_{1}$, with multiplicity $(2 g-2)(d-1)$ !. (The multiplicities are easily explained: $2 g-2+2 d$ is the degree of the ramification divisor, $2 g-2$ is the degree of the canonical divisor, and the factorials arise since the remaining 
points are ordered.) Equating the two expressions, one obtains a kappa relation in codimension $g-2 d+2$. We find therefore one kappa relation in codimension $c$ for each $c \leq g-2$.

Ionel [34] has proved that the first $\lfloor g / 3\rfloor$ kappa classes $\kappa_{1}, \ldots, \kappa_{\lfloor g / 3\rfloor}$ generate $R^{*}\left(M_{g}\right)$ using these relations. Earlier, Morita [47] had obtained the analogous result for $R H^{*}\left(M_{g}\right)$ using a completely different set of relations.

One could also consider the case $d=1$. The locus in $C_{g}$ is empty, so we find a relation on $C_{g}$ :

$$
0=\Delta_{1, g}\left(c_{t}\left(\mathbb{F}_{1}\right) / c_{t}(\mathbb{E})\right)=\Delta_{g, 1}\left(c_{t}\left(\mathbb{E}^{\vee}\right) / c_{t}\left(\mathbb{F}_{1}^{\vee}\right)\right)=(-1)^{g}\left(c_{t}(\mathbb{E}) / c_{t}\left(\mathbb{F}_{1}\right)\right)_{g} .
$$

(The subscript $g$ on the right means taking the coefficient of $t^{g}$.) This is exactly the same relation as Mumford's relation discussed above.

As a second example, consider the residual linear system of a $g_{2}^{1}$, namely, a $g_{2 g-4}^{g-2}$, obtained from $g-2$ copies of the $g_{2}^{1}$ (the curve is necessarily hyperelliptic and the $g_{2}^{1}$ is unique). The expected codimension equals the actual codimension, since equality holds for a linear system if and only if it holds for the residual system. Porteous's formula gives the class of the locus of divisors moving in a $g_{2 g-4}^{g-2}$ as

$$
\Delta_{g-2,2}\left(c_{t}\left(\mathbb{F}_{2 g-4}\right) / c_{t}(\mathbb{E})\right)=\left(c_{g-2}^{2}-c_{g-1} c_{g-3}\right)\left(\mathbb{F}_{2 g-4}-\mathbb{E}\right) .
$$

(The difference is taken in the Grothendieck group; this notation is sometimes more convenient.)

The locus has $(g-2)$-dimensional fibers over the hyperelliptic locus $H_{g}$ in $M_{g}$ (of codimension $g-2$ ). To obtain relations, we can cut with $c<g-2$ tautological divisors and push forward, to obtain a relation in codimension $c$; or we cut with $g-2$ divisors and obtain the class of $H_{g}$ with some multiplicity. It is not hard to check that the number of essentially different ways in which one can cut with $c$ nonoverlapping diagonals equals the number $p(c)$ of partitions of $c$. Thus, this choice gives $p(c)$ kappa relations in codimension $c$ for every $c<g-2$, and $p(g-2)-1$ relations in codimension $g-2$. As we will see later, this method may produce all kappa relations in codimensions up to $g-2$. As far as I know, it has not been studied in detail.

As a third example, consider the divisors moving in a $g_{2 g-1}^{g}$. The locus is empty, and Porteous's formula gives the relation

$$
c_{g}\left(\mathbb{F}_{2 g-1}-\mathbb{E}\right)=0
$$

on $C_{g}^{2 g-1}$. The bundle map $\mathbb{E} \rightarrow \mathbb{F}_{2 g-1}$ is therefore injective with quotient bundle of rank $g-1$, so that $c_{j}\left(\mathbb{F}_{2 g-1}-\mathbb{E}\right)=0$ for all $j \geq g$. Analogously, $c_{j}\left(\mathbb{F}_{d}-\mathbb{E}\right)=0$ for $d \geq 2 g-1$ and $j \geq d-g+1$.

Let us concentrate on the relation $c_{g}\left(\mathbb{F}_{2 g-1}-\mathbb{E}\right)=0$ of codimension $g$ on $C_{g}^{2 g-1}$. If we push it forward to $M_{g}$, we land in negative codimension, so that the obtained relation is necessarily trivial. To have a chance of obtaining nontrivial relations on $M_{g}$, we should cut with $c+g-1$ tautological divisors for a relation in codimension $c$. As it turns out, this method produces many nontrivial relations. Moreover, this class of relations has several good properties, which makes it possible on the one hand to analyse them theoretically and on the other hand to compute them for quite large genus. In fact, the results of computing these relations for $g \leq 15$ were 
to me so convincing that I conjectured (in 1993) a precise description of $R^{*}\left(M_{g}\right)$. I will now recall this conjecture [10].

The first part of the conjecture is that $R^{*}\left(M_{g}\right)$ is a Gorenstein ring with socle in degree $g-2$. This means that $R^{j}\left(M_{g}\right)=0$ for $j>g-2$, that $R^{g-2}\left(M_{g}\right)$ is isomorphic to $\mathbb{Q}$, and that the natural pairing

$$
R^{i}\left(M_{g}\right) \times R^{g-2-i}\left(M_{g}\right) \rightarrow R^{g-2}\left(M_{g}\right)
$$

is supposed to be perfect.

Secondly, I conjectured that $R^{*}\left(M_{g}\right)$ is generated by $\kappa_{1}, \ldots, \kappa_{\lfloor g / 3\rfloor}$ and that there are no relations in degrees $\leq g / 3$.

Thirdly, I gave explicit formulas for the proportionalities in degree $g-2$. I will not recall these formulas here, but note that they determine $R^{*}\left(M_{g}\right)$ completely if the first part of the conjecture is true.

Thanks to the work of many people, large parts of the conjecture are now proved. As to the first part, Looijenga $[\mathbf{4 3}]$ proved the vanishing in degrees greater than $g-2$. He also proved that $R^{g-2}\left(M_{g}\right)$ is at most one-dimensional. I proved $[10,14]$ that $\kappa_{g-2}$ is nonzero, so $R^{g-2}\left(M_{g}\right)$ is isomorphic to $\mathbb{Q}$. As to the second part, the generation statement was proved by Morita for $R H^{*}\left(M_{g}\right)$ and by Ionel for $R^{*}\left(M_{g}\right)$, as already discussed. From the work of Boldsen [5], it follows that there are no relations in degrees $\leq g / 3$, so the second part of the conjecture is completely proved. ${ }^{1}$ The third part is completely proved, and there are in fact three proofs: by Givental [26], following earlier work of Eguchi-Hori-Xiong [9] and Getzler-Pandharipande [25]; by Liu and Xu [41]; and by Buryak and Shadrin [6].

Finally, the complete conjecture has been proved for $g \leq 23$. For all these genera, the method of the third example above has produced sufficiently many relations (by cutting $c_{g}\left(\mathbb{F}_{2 g-1}-\mathbb{E}\right)$ with non-overlapping diagonals and pushing down to $M_{g}$ ).

The fact that $R^{g-2}\left(M_{g}\right)$ is one-dimensional, generated by $\kappa_{g-2}$, and that the third part of the conjecture is proved, means that it makes sense to talk about the Gorenstein quotient $G^{*}\left(M_{g}\right)$ of $R^{*}\left(M_{g}\right)$. This is one-dimensional in degree $g-2$ and is the quotient of $R^{*}\left(M_{g}\right)$ by the homogeneous ideal generated by all classes of pure degree that have zero pairing with all classes of the complementary degree.

The ring $G^{*}\left(M_{g}\right)$ can be studied independently from $R^{*}\left(M_{g}\right)$. This will of course not prove the Gorenstein conjecture. The point is rather to try to make the kappa relations in the Gorenstein quotient explicit.

Zagier and I have studied $G^{*}\left(M_{g}\right)$ in considerable detail. A first question is what the dimensions of the graded pieces are. Let $q(g, k)$ denote the dimension of $G^{k}\left(M_{g}\right)$. Then $q(g, k)=q(g, g-2-k)$ by definition, and $q(g, k) \leq p(k)$ (the number of partitions of $k$ ), with equality conjectured for $k \leq g / 3$. We would like to know $p(k)-q(g, k)$ for $g / 3<k \leq\lfloor(g-2) / 2\rfloor$. These values are known for $g \leq 23$ by the truth of the conjecture and can be computed for further genera by linear algebra. One observes a remarkable property: $p(k)-q(g, k)$ turns out to be a function of $3 k-g-1$. Let us assume that this is indeed the case, so that

$$
a(3 k-g-1)=p(k)-q(g, k)
$$

${ }^{1}$ I am grateful to Randal-Williams for explaining to me how this follows when the degree equals $g / 3$. 
for an unknown function $a$. One can compute several values of the function $a$ :

\begin{tabular}{c||c|c|c|c|c|c|c|c|c|c}
$\ell$ & 0 & 1 & 2 & 3 & 4 & 5 & 6 & 7 & 8 & 9 \\
\hline$a(\ell)$ & 1 & 1 & 2 & 3 & 5 & 6 & 10 & 13 & 18 & 24
\end{tabular}

Based on these values, Zagier and I made the following guess: $a(m)$ equals the number of partitions of $m$ in parts of the form

$$
1,2,3,4,6,7,9,10,12,13,15, \ldots,
$$

that is, the only part congruent to 2 modulo 3 that can be used is the part 2 . Fairly recently, Liu and $\mathrm{Xu}[\mathbf{4 2}]$ computed five more values of the function $a$ (note that their $a(n)$ equals our $a(n-1))$. We see that the guess that Zagier and I made is compatible with these new values.

However, it may be more important to study the actual kappa relations in $G^{*}\left(M_{g}\right)$. The form of the (conjecturally) unique relation in codimension $k$ and genus $3 k-1$ was found, and later proved, long ago by Zagier and me (see [10], pp. 124-125). Later, we also found the form of the relation in codimension $k$ and genus $3 k-2$. These relations were also derived by Morita [47] and Ionel [34].

Continuing this work, Zagier and I wanted to find explicit expressions for the relations in 'the first half' of $G^{*}\left(M_{g}\right)$, i.e., in codimensions $k \leq(g-2) / 2$, where the numbers of relations are directly governed by the function $a$ (instead of by duality). Approximately ten years ago, we obtained the following result. Let

$$
\mathbf{p}=\left\{p_{1}, p_{3}, p_{4}, p_{6}, p_{7}, p_{9}, p_{10}, \ldots\right\}
$$

be a collection of variables indexed by the positive integers not congruent to 2 modulo 3. Let $\Psi(t, \mathbf{p})$ be the following formal power series:

$$
\Psi(t, \mathbf{p})=\sum_{i=0}^{\infty} t^{i} p_{3 i} \sum_{j=0}^{\infty} \frac{(6 j) !}{(3 j) !(2 j) !} t^{j}+\sum_{i=0}^{\infty} t^{i} p_{3 i+1} \sum_{j=0}^{\infty} \frac{(6 j) !}{(3 j) !(2 j) !} \frac{6 j+1}{6 j-1} t^{j},
$$

where $p_{0}:=1$. Define rational numbers $C_{r}(\sigma)$, for $\sigma$ any partition (of $|\sigma|$ ) with parts not congruent to 2 modulo 3 , by the formula

$$
\log (\Psi(t, \mathbf{p}))=\sum_{\sigma} \sum_{r=0}^{\infty} C_{r}(\sigma) t^{r} \mathbf{p}^{\sigma}
$$

where $\mathbf{p}^{\sigma}$ denotes $p_{1}^{a_{1}} p_{3}^{a_{3}} p_{4}^{a_{4}} \ldots$ if $\sigma$ is the partition $\left[1^{a_{1}} 3^{a_{3}} 4^{a_{4}} \ldots\right]$. Put

$$
\gamma=\sum_{\sigma} \sum_{r=0}^{\infty} C_{r}(\sigma) \kappa_{r} t^{r} \mathbf{p}^{\sigma}
$$

then the relation

$$
[\exp (-\gamma)]_{t^{r}} \mathbf{p}^{\sigma}=0
$$

holds in the Gorenstein quotient when $g-1+|\sigma|<3 r$ and $g \equiv r+|\sigma|+1(\bmod 2)$. (Of course, $\kappa_{0}=2 g-2$.)

Let me call these relations (in the Gorenstein quotient) the FZ-relations for brevity. Observe that we get the expected number of relations in every codimension less than or equal to $\lfloor(g-2) / 2\rfloor$, although we didn't prove that the obtained relations in such a codimension are independent. Our goal was precisely to understand the relations until the middle; just by looking at the numbers of relations, it is clear that the FZ-relations cannot give all relations in $G^{*}\left(M_{g}\right)$ for $g$ large enough (more precisely, for odd $g \geq 25$ and even $g \geq 30$ ). 
Nevertheless, one can study the actual rank of the FZ-relations, and Pandharipande and I looked at this a few years ago. The first result is that the FZ-relations give all relations for $g \leq 23$. For $g=24$, however, one relation (in codimension 12) in the Gorenstein quotient is missing: the $41 \mathrm{FZ}$-relations in degree 12 admit an unexpected syzygy and span only a 40-dimensional space of relations. The quotient by the FZ-relations has rank $p(12)-40=37$ in degree 12 , whereas it has rank $p(10)-a(5)=42-6=36$ in degree 10 . Similarly, the quotient by the FZrelations differs from the Gorenstein quotient by one relation in genera 25 and 26 (in codimension 12 resp. 13); for $g=25$, this is the optimal outcome, but for $g=26$, there is another unexpected syzygy.

The fact that the FZ-relations don't give all relations in the Gorenstein quotient is not surprising (as just mentioned). It is surprising that there are unforced syzygies between the FZ-relations in certain degrees, but on the other hand, in higher degrees there are very many syzygies between the FZ-relations. In any case, one wonders what can be said about the actual relations in $R^{*}\left(M_{g}\right)$.

Let us study the case of genus $g=24$ and codimension $c=12$. If we use the method of the third example above and restrict to cutting $c_{g}\left(\mathbb{F}_{2 g-1}-\mathbb{E}\right)$ with non-overlapping diagonals before pushing down to $M_{g}$, then the (a priori) distinct possibilities correspond to the partitions of $2 g-1=47$ of length equal to $g-c=12$. If the partition contains a part equal to 1 , the relation is trivial (exercise), so we consider only partitions with all parts at least equal to 2 . There are 1116 such partitions. So far, I have computed approximately 250 of the corresponding relations. The space spanned by these relations coincides exactly with the 40dimensional space spanned by the 41 FZ-relations.

In the other codimensions in genus 24, the 'diagonal' method produces all relations. In other words, the spaces of diagonal and FZ-relations coincide for those codimensions. It is not known what happens in codimension 12, but it is hard to believe that the diagonal method will produce the missing Gorenstein relation. In genera 25 and 26, the situation is similar at the moment, although not as many computations have been done. Perhaps the diagonal method will always produce exactly the space of FZ-relations.

Recently, Pandharipande and Pixton [53] proved the strong result that the FZrelations are actual relations in $R^{*}\left(M_{g}\right)$ (and not just in $G^{*}\left(M_{g}\right)$ ). The geometric origin of the relations used in the proof of this result is the moduli space of stable quotients, introduced and studied by Marian, Oprea, and Pandharipande [45]. The method of virtual localization on this space produces a wealth of relations. A subset of these relations could be worked out to some extent; and for a smaller subset, the relations could be made even more explicit. Finally, Pandharipande and Pixton obtained the striking result that this last subset of explicit relations is equivalent to the set of FZ-relations.

The current state of affairs allows several interpretations. Perhaps $R^{*}\left(M_{g}\right)$ is not Gorenstein for $g \geq 24$. One would then like to have a proof, at least in some cases, that a 'missing' Gorenstein relation is not an actual relation. On the other hand, perhaps $R^{*}\left(M_{g}\right)$ is Gorenstein after all. Then a new source of relations would seem to be necessary (and it should be possible to compute or analyse these relations for $g \geq 24)$. Finally, the intriguing possibility remains that $R^{*}\left(M_{g}\right)$ and $R H^{*}\left(M_{g}\right)$ differ. 


\section{Exercises}

1. Let $V$ be a vector bundle of rank $r$. Review how one expresses the components $c h_{j}(V)$ of the Chern character in terms of the Chern classes $c_{i}(V)$. Use this to prove the equivalence of

$$
\operatorname{ch}_{2 i}(V)=0 \quad(\forall i \geq 1)
$$

and

$$
c_{t}\left(V^{\vee}\right)=c_{t}(V)^{-1}
$$

Next, assume that these equivalent statements hold. Prove that

$$
c h_{i}(V)=0
$$

for all $i \geq 2 r$. Show also that $c h_{2 r-1}(V)$ and $c_{r}(V) c_{r-1}(V)$ are multiples of each other. Finally, apply the results above to the Hodge bundle $\mathbb{E}$ of rank $g$ on $M_{g}$ and derive in particular the formula for the total Chern class given in the text.

2. The Bernoulli numbers $B_{2 i}$ are defined by $t /\left(e^{t}-1\right)=\sum_{i=0}^{\infty} B_{i} t^{i} /(i$ !). They satisfy several well-known congruences; in particular, for $i>0$,

$$
B_{2 i}+\sum_{\substack{p \text { prime: } \\(p-1) \mid 2 i}} \frac{1}{p}
$$

is an integer. This implies immediately

$$
v_{2}\left(B_{2 i}\right)=-1
$$

for $i>0$. (Here $v_{2}: \mathbb{Q} \backslash\{0\} \rightarrow \mathbb{Z}$ is the standard 2-adic valuation.) Prove Mumford's result that $\kappa_{1}, \ldots, \kappa_{g-2}$ generate $R^{*}\left(M_{g}\right)$ using the relations mentioned in the text. The only property of the Bernoulli numbers that you will need is their 2-adic valuation given above. (Distinguish between $g$ even and odd. The case $g$ odd is a little harder.)

3. Prove the formula for $c\left(\mathbb{F}_{d}\right)$ given in the text, for example as follows.

(a) Let $\Sigma_{k}$ denote the subscheme of the universal curve $\pi: U \rightarrow C_{g}^{d}$ that is the (formal) sum of the images of the first $k$ sections $s_{1}, \ldots, s_{k}$ of $\pi$. Prove that there is an exact sequence

$$
0 \rightarrow \mathcal{O}_{s_{d}}\left(-s_{1}-\cdots-s_{d-1}\right) \rightarrow \mathcal{O}_{\Sigma_{d}} \rightarrow \mathcal{O}_{\Sigma_{d-1}} \rightarrow 0 .
$$

(b) Tensor the exact sequence with $\omega_{\pi}$ and prove that the sheaf on the left is the push-forward via $s_{d}$ of the line bundle with first Chern class $K_{d}-\Delta_{d}$.

(c) Obtain the result by applying $\pi_{*}$ and using induction.

4. Show that the results of Boldsen in [5] imply that there are no relations between the kappa classes in (algebraic) degree $k<g / 3$. With more work, show that this also holds in degree $g / 3$.

5. Using the ' $g_{d}^{r}$-method' as discussed in the lectures (in any of the three incarnations in the notes, or in another one), derive a nontrivial relation between the kappa classes (in some genus).

6. Same problem as above, but now with the further condition that the degree $k$ of the relation satisfies $g / 3<k \leq g-2$ (so that $g \geq 4$ ).

7. Well-known classical formulas for plane curves (typically derived by studying the dual plane curve in the dual projective plane) say that a general (smooth) plane curve of degree $d$ over the complex numbers (which has genus $g=\frac{1}{2}(d-1)(d-2)$ ) has $3 d(d-2)$ inflection points (a.k.a. flexes - points where the tangent line has 
contact of order 3$)$ and $\frac{1}{2} d(d-2)\left(d^{2}-9\right)$ bitangents (lines that are tangent in two distinct points).

Verify this for $d=4$ by using the ' $g_{d}^{r}$-method' for $r=2$ and $d=4$ (hence $g=3$ ). (Cut the class in $C_{3}^{4}$ with two appropriate diagonal divisors and push it down to $M_{3}$. The calculations simplify considerably by doing some of the push-downs in a clever way. For example, in the flex case, one can use a bundle on $C_{3}^{2}$ with total Chern class $\left(1+K_{1}\right)\left(1+2 K_{1}\right)\left(1+3 K_{1}\right)\left(1+K_{2}-3 D_{1,2}\right)$.)

If you feel courageous, compute the class of the locus of plane quintics in $M_{6}$ in two ways by the same method, and derive a relation in $R^{3}\left(M_{6}\right)$ as a result.

Tacitly, I have been assuming in this exercise that the conditions in Porteous's formula are fulfilled. If you are an algebraic geometer, verify this. 
LECTURE 2

\section{The tautological rings of $\bar{M}_{g, n}$ and of some natural partial compactifications of $M_{g, n}$}

Let $M_{g, n}$ denote the moduli space of $n$-pointed nonsingular curves of genus $g$ (where $g$ and $n$ are nonnegative integers with $2 g-2+n>0$ ). The $n$ points are distinct and ordered. As is well-known $[\mathbf{8}, \mathbf{3 7}, \mathbf{3 8}]$, this moduli space admits a natural compactification: the Deligne-Mumford-Knudsen moduli space $\bar{M}_{g, n}$ of stable $n$-pointed curves of genus $g$. The curves are allowed to have ordinary double points as singularities, but are required to be connected. The $n$ ordered points remain distinct and nonsingular. The $n$-pointed curves must have finite automorphism group; this is the stability condition.

The space $\bar{M}_{g, n}$ admits a natural stratification, by topological type: a stratum consists of all the $n$-pointed curves homeomorphic (as pointed curves with the complex topology) to a given one. (For a detailed discussion of the material contained in these introductory paragraphs, I refer to [28], Appendix.) The topological type is conveniently encoded in the dual graph, with $n$ ordered legs and a genus function on the set $V$ of vertices (which correspond to the normalizations of the irreducible components). The stratum is isomorphic to the quotient of the product $\prod_{v \in V} M_{g(v), n(v)}$ by the automorphism group of the dual graph. Here $n(v)$ equals the number of points on the smooth curve corresponding to $v$ that map to a marked point or a node of the stable curve. (The stability condition is that $2 g(v)-2+n(v)>0$ for all $v \in V$.) The quotient of $\prod_{v \in V} \bar{M}_{g(v), n(v)}$ by the automorphism group of the graph is the normalization of the closure of the stratum (which is a union of strata).

How should the tautological ring $R^{*}\left(\bar{M}_{g, n}\right)$ of $\bar{M}_{g, n}$ be defined? We certainly want the classes of the closures of the strata to be tautological. Considering the natural map from $\prod_{v \in V} \bar{M}_{g(v), n(v)}$ to $\bar{M}_{g, n}$ with image the closure of a stratum, we also want the push-forward of a product of tautological classes on the factors to be tautological. The most natural way of defining the $\kappa$-classes on $\bar{M}_{g, n}$ was given by Arbarello and Cornalba [1]:

$$
\kappa_{i}=\pi_{*}\left(\psi_{n+1}^{i+1}\right)
$$

where $\pi: \bar{M}_{g, n+1} \rightarrow \bar{M}_{g, n}$ is the map given by forgetting the $(n+1)$ st point and stabilizing and $\psi_{j}$ is the first Chern class of the cotangent line bundle at the $j$ th point. (If the $(n+1)$ st point lies on a 3 -pointed curve of genus 0 , the curve becomes unstable after forgetting the point. The component needs to be contracted; this is the process of stabilization.)

Continuing with this kind of considerations, one arrives eventually at a definition of $R^{*}\left(\bar{M}_{g, n}\right)$, but it seems somewhat ad hoc. A perhaps more natural definition 
was given by Pandharipande and myself: we define the system

$$
\left\{R^{*}\left(\bar{M}_{g, n}\right)\right\}_{(g, n)}
$$

as the system of minimal $\mathbb{Q}$-subalgebras of the rational Chow rings $A^{*}\left(\bar{M}_{g, n}\right)$ closed under push-forward via all maps forgetting markings and all gluing maps (see [16], pp. 13-14). One shows that the psi and kappa classes are tautological, and therefore also the push-forward of a product of monomials in the psi and kappa classes on a product of compact moduli spaces as above. In fact, as shown in [28], Prop. 11, these classes generate $R^{*}\left(\bar{M}_{g, n}\right)$ additively. The system of tautological rings is also closed under pull-back via the forgetting and gluing maps. It follows from Mumford's computation of the Chern character of the Hodge bundle $[\mathbf{4 8}, \S 5]$ that the lambda classes are tautological on $\bar{M}_{g}$.

Let $U$ be an open subvariety of $\bar{M}_{g, n}$. We use the surjectivity of the restriction maps $A^{k}\left(\bar{M}_{g, n}\right) \rightarrow A^{k}(U)$ (cf. [19], §1.8) to define the tautological ring $R^{*}(U)$ as the image of $R^{*}\left(\bar{M}_{g, n}\right)$.

For $M_{g}$, this agrees with the earlier definition. (An independent motivation is provided by the Madsen-Weiss theorem describing the stable cohomology. Recall that Harer [30] has proved that the cohomology groups $H^{k}\left(M_{g}\right)$ stabilize as $g \rightarrow \infty$. The resulting algebra is called the stable cohomology and may be denoted $H^{*}\left(M_{\infty}, \mathbb{Q}\right)$. Madsen and Weiss $[\mathbf{4 4}]$ prove that $H^{*}\left(M_{\infty}, \mathbb{Q}\right)$ equals the free polynomial algebra in the kappa classes.) Note that there is in general no guarantee that the kernel of the map $R^{k}\left(\bar{M}_{g, n}\right) \rightarrow R^{k}(U)$ is spanned by tautological classes supported on the complement of $U$.

The partial compactifications $U$ of $M_{g, n}$ in whose tautological rings we are most interested are the moduli space $M_{g, n}^{c}$ of curves of compact type, the complement of the divisor $\Delta_{i r r}$ of irreducible singular curves and their degenerations, and (for $g \geq 2$ ) the moduli space $M_{g, n}^{r t}$ of curves with rational tails, the inverse image of $M_{g}$ for the map $\bar{M}_{g, n} \rightarrow \bar{M}_{g}$. The work of Graber and Vakil [29] shows that other partial compactifications are important as well: they prove that a class in $R^{i}\left(\bar{M}_{g, n}\right)$ vanishes when restricted to the open set consisting of strata parameterizing curves with at most $i-g$ components of geometric genus zero.

It follows that $R^{*}\left(M_{g, n}\right)$ vanishes in positive degrees $\geq g$, that $R^{*}\left(M_{g, n}^{r t}\right)$ vanishes in degrees $\geq g-1+n$, and that $R^{*}\left(M_{g, n}^{c}\right)$ vanishes in degrees $\geq 2 g-2+n$. With more work, one shows that $R^{g-2+n}\left(M_{g, n}^{r t}\right), R^{2 g-3+n}\left(M_{g, n}^{c}\right)$ and $R^{3 g-3+n}\left(\bar{M}_{g, n}\right)$ are one-dimensional (cf. [29] and [16]). The classes $\lambda_{g}$ and $\lambda_{g} \lambda_{g-1}$ play a crucial role in the nonvanishing statement: they vanish on the complement of $M_{g, n}^{c}$, respectively $M_{g, n}^{r t}$.

A fundamental result for the study of the tautological rings is Witten's conjecture [58]. It completely determines the intersection numbers (or integrals) of psi classes on all the moduli spaces $\bar{M}_{g, n}$. It was first proved by Kontsevich [39] and there are by now several proofs (e.g., by Mirzakhani [46] and OkounkovPandharipande [49]; Mirzakhani's proof is the subject of Wolpert's lectures and notes $[\mathbf{5 9}])$.

The psi integrals on $\bar{M}_{g, n}$ determine the kappa integrals on $\bar{M}_{g}$, and vice versa. The recipes for both transitions are relatively simple (cf. $[\mathbf{1}, \mathbf{3 5}])$. In fact, the psi integrals determine the full intersection pairing on $R^{*}\left(\bar{M}_{g, n}\right)$ (cf. [28], Props. 10, 11). The situation is entirely analogous for the psi integrals against $\lambda_{g}$ or $\lambda_{g} \lambda_{g-1}$; they determine the intersection pairing on $R^{*}\left(M_{g, n}^{c}\right)$, respectively $R^{*}\left(M_{g, n}^{r t}\right)$. 
The psi integrals against $\lambda_{g}$ satisfy amazingly simple proportionalities:

$$
\int_{\bar{M}_{g, n}} \psi_{1}^{a_{1}} \cdots \psi_{n}^{a_{n}} \lambda_{g}=\left(\begin{array}{c}
2 g-3+n \\
a_{1}, \ldots, a_{n}
\end{array}\right) \int_{\bar{M}_{g, 1}} \psi_{1}^{2 g-2} \lambda_{g},
$$

where the integral on the right side equals 1 by definition for $g=0$. The proportionalities were derived by Getzler and Pandharipande [25] from the Virasoro conjecture of Eguchi, Hori, and Xiong [9]. They were first proved in [15]; see also $[40,27]$. The not quite as simple proportionalities satisfied by the psi integrals against $\lambda_{g} \lambda_{g-1}$ are part of my conjecture describing $R^{*}\left(M_{g}\right)$. They were proved directly by Liu and $\mathrm{Xu}[4 \mathbf{4 1}$ and later by Buryak and Shadrin [6]. A more roundabout proof for both sets of proportionalities is obtained from Givental's proof of the Virasoro conjecture for projective spaces [26]. The kappa integrals against $\lambda_{g} \lambda_{g-1}$ are not nearly as simple; yet they govern the Gorenstein quotient $G^{*}\left(M_{g}\right)$.

In analogy with the conjecture for $R^{*}\left(M_{g}\right)$, it has been speculated that the rings $R^{*}\left(\bar{M}_{g, n}\right), R^{*}\left(M_{g, n}^{c}\right)$ and $R^{*}\left(M_{g, n}^{r t}\right)$ all are Gorenstein. It has long been known that $R^{*}\left(\bar{M}_{0, n}\right)$ is Gorenstein $[\mathbf{3 6}]$. Recently, Tavakol [55, 56] proved that $R^{*}\left(M_{1, n}^{c}\right)$ and $R^{*}\left(M_{2, n}^{r t}\right)$ are Gorenstein. ${ }^{1}$ Besides this, the Gorenstein property is known in only a handful of cases. One of the main difficulties (at least in the case of $\bar{M}_{g, n}$ ) is the large number of tautological classes. Another one is that the undetermined boundary terms of a tautological relation on an open set are rarely known to be tautological; Tavakol's codimension 3 relation on $R^{*}\left(M_{2,6}^{r t}\right)$ provides a current example. However, see [53] for an encouraging exception.

There is considerable room for further exploration of these speculations. Yang [60] has computed the dimensions of the graded pieces of the Gorenstein quotients in quite a few cases. To prove that one of the rings is Gorenstein, one needs to produce the required number of tautological relations. See [12] for a discussion of the conjectures and the relation to a conjecture of Hain and Looijenga. Pandharipande [51] has obtained strong results on the kappa subrings of $R^{*}\left(M_{g, n}^{c}\right)$ (see also [52]). Finally, Cavalieri and Yang [7] show that the Gorenstein property fails for the tautological rings of certain partial compactifications in between $M_{g, n}^{c}$ (respectively $\left.M_{g, n}^{r t}\right)$ and $\bar{M}_{g, n}$, while the top graded pieces are one-dimensional.

Many tautological relations, i.e., between decorated strata classes, have immediate implications for Gromov-Witten theory. Well-known examples are the relations on $\bar{M}_{0,4}$, Getzler's relation on $\bar{M}_{1,4}$, and the Belorousski-Pandharipande relation on $\bar{M}_{2,3}(\mathrm{cf}$. $[\mathbf{2 1}, \mathbf{5 0}, \mathbf{3}])$. Tommasi $[\mathbf{5 7}]$ has found a codimension 3 relation between tautological cohomology classes on $\bar{M}_{3,2}$. It is not known whether this relation holds in $R^{3}\left(\bar{M}_{3,2}\right)$. Pandharipande and the author found a codimension 3 relation in $R^{*}\left(\bar{M}_{4}\right)$, predicted on $M_{4}^{c}$ by the Gorenstein conjecture; Yang [60] computed its boundary terms. It is a priori possible that nontrivial relations hold between the push-forwards along a gluing map of decorated strata classes that are not push-forwards of relations. Such relations would be of considerable interest.

The length of a partition is its number of parts. There exists a standard correspondence between the irreducible representations of the symmetric group $\Sigma_{n}$ and the partitions of $n$ (see, e.g., [20]; the partition $[n]$ corresponds to the trivial representation and the partition $\left[1^{n}\right]$ to the alternating one). In $[\mathbf{1 7}]$, Pandharipande

\footnotetext{
${ }^{1}$ Note added in proof: Petersen [54] has proved that $R^{*}\left(\bar{M}_{1, n}\right)$ is Gorenstein for all $n \geq 1$. Even more recently, Yin [61] has proved that $R^{*}\left(M_{g, 1}\right)$ is Gorenstein for $g \leq 19$. He conjectures that $R^{*}\left(M_{g}\right)$ and $R^{*}\left(M_{g, 1}\right)$ are not Gorenstein in general.
} 
and the author prove certain length bounds on the partitions of $n$ corresponding to the irreducible representations of $\Sigma_{n}$ occurring in $R^{*}\left(\bar{M}_{g, n}\right)$. Perhaps the most interesting result is the following:

$$
\ell\left(R^{k}\left(\bar{M}_{g, n}\right)\right) \leq \min \left(k+1,3 g-2+n-k,\left\lfloor\frac{2 g-1+n}{2}\right\rfloor\right),
$$

where the left side denotes the maximum of the lengths. Using this bound, we have with near certainty established (loc. cit., §3) the existence of lots of non-tautological cohomology classes of Tate type on $\bar{M}_{2,21}$; this relies on a conjectural description (proved to a large extent and supported by extensive point counting data) of the entire cohomology of $\bar{M}_{2, n}$ found by van der Geer and the author (cf. [13]). In fact, in $\S 5$ of [17], explicit non-tautological algebraic cohomology classes are found on $\bar{M}_{2,21}$, the existence of which implies the non-tautologicality of the explicit classes on $\bar{M}_{2,22}$ found earlier by Graber and Pandharipande [28].

With all the interest for the tautological algebras, it is easy to forget that there are in general many more cohomology classes than just the tautological ones. Only in finitely many cases beyond genus zero will the entire cohomology be tautological, it would seem. One should expect odd cohomology, or cohomology not of $(p, p)$ type, or (on a non-compact moduli space) non-pure cohomology of $(p, p)$-type; and, probably slightly less often, algebraic classes which are not tautological.

A successful method for obtaining a lot of information about the entire cohomology of moduli spaces of curves and abelian varieties, used in joint work with Bergström and van der Geer, has been to count their numbers of points over finite fields. For a survey, see $\S \S 2$ and 3 of [17]. For genus 1, one finds cohomology related to elliptic cusp forms; Getzler $[\mathbf{2 2}, \mathbf{2 3}]$ has shown how the classical Eichler-Shimura theory can be used to determine the cohomology of $\bar{M}_{1, n}$. The generalization by Faltings and Chai [18] of Eichler-Shimura theory gives strong results on the cohomology of irreducible symplectic local systems on the moduli space $A_{g}$ of principally polarized abelian varieties of dimension $g$. The same holds for the theory of automorphic forms and representations, but it is not easy to obtain explicit formulas. Explicit conjectural formulas are now available for $g=2$ (see [13]) and $g=3$ (see [4]). The former is proved in the case of a local system corresponding to a regular weight. The formulas are in terms of Galois representations (or Hodge structures) associated to Siegel cusp forms of degree $g$ and products or Tate twists of Galois representations associated to Siegel cusp forms of lower degree. This leads to formulas for the pull-backs of the local systems to $M_{2}$, and from there (as pointed out by Getzler) to the $\Sigma_{n}$-equivariant cohomology of $M_{2, n}$, and (by the work of Getzler and Kapranov [24]) to the $\Sigma_{n}$-equivariant cohomology of $\bar{M}_{2, n}$. The symmetric and exterior squares of the Galois representations associated to elliptic cusp forms occur now as well. For genus 3 , the formula for $A_{3}$ gives at best half of the answer for $M_{3}$, since the map of stacks $M_{3} \rightarrow A_{3}$ is $2: 1$ onto its image, ramified along the hyperelliptic locus. We have proved that new Galois representations occur, not expressible in terms of the representations associated to Siegel cusp forms. These Galois representations are so far completely mysterious, but appear to be associated to Teichmüller modular forms (vector-valued in general). Scalar-valued Teichmüller modular forms have been studied in detail by Ichikawa (e.g., $[\mathbf{3 1}, \mathbf{3 2}, \mathbf{3 3}])$. 


\section{Exercises}

1. Prove that the undecorated strata classes additively generate $R^{*}\left(\bar{M}_{g, n}\right)$ for $g \leq 1$. Conclude that $R^{*}\left(\bar{M}_{0, n}\right)$ is multiplicatively generated by divisor classes. Does this hold for $g=1$ ?

2. Study $R^{*}\left(\bar{M}_{g, n}\right)$ in some cases (with $2 g-2+n$ small) where it is multiplicatively generated by divisor classes, for example with the program $\mathbf{M g n F}$, described in [11] and available from http://math.stanford.edu/ vakil/programs/index.html .

3. Find a case in Yang's tables [60] where the Gorenstein property is not yet known and try to prove (or disprove) that it holds. 



\section{Bibliography}

[1] E. Arbarello and M. Cornalba, Combinatorial and algebro-geometric cohomology classes on the moduli spaces of curves, J. Algebraic Geom. 5 (1996), 705-749.

[2] E. Arbarello, M. Cornalba, P.A. Griffiths, and J. Harris, Geometry of algebraic curves. Vol. I. Grundlehren der Mathematischen Wissenschaften, 267. Springer-Verlag, New York, 1985.

[3] P. Belorousski and R. Pandharipande, A descendent relation in genus 2. Ann. Scuola Norm. Sup. Pisa Cl. Sci. (4) 29 (2000), no. 1, 171-191.

[4] J. Bergström, C. Faber, and G. van der Geer, Siegel modular forms of degree three and the cohomology of local systems. Preprint, arXiv:1108.3731.

[5] S. Boldsen, Improved homological stability for the mapping class group with integral or twisted coefficients. Preprint, arXiv:0904.3269.

[6] A. Buryak and S. Shadrin, A new proof of Faber's intersection number conjecture. Adv. Math. 228 (2011), no. 1, 22-42.

[7] R. Cavalieri and S. Yang, Tautological pairings on moduli spaces of curves. Proc. Amer. Math. Soc. 139 (2011), no. 1, 51-62.

[8] P. Deligne and D. Mumford, The irreducibility of the space of curves of given genus. Inst. Hautes Études Sci. Publ. Math. No. 361969 75-109.

[9] T. Eguchi, K. Hori, and C.-S. Xiong, Quantum cohomology and Virasoro algebra. Phys. Lett. B 402 (1997), no. 1-2, 71-80.

[10] C. Faber, A conjectural description of the tautological ring of the moduli space of curves, in Moduli of Curves and Abelian Varieties (The Dutch Intercity Seminar on Moduli) (C. Faber and E. Looijenga, eds.), 109-129, Aspects of Mathematics E 33, Vieweg, Wiesbaden 1999.

[11] C. Faber, Algorithms for computing intersection numbers on moduli spaces of curves, with an application to the class of the locus of Jacobians. New trends in algebraic geometry (Warwick, 1996), 93-109, London Math. Soc. Lecture Note Ser., 264, Cambridge Univ. Press, Cambridge, 1999.

[12] C. Faber, A remark on a conjecture of Hain and Looijenga. Preprint, arXiv:0812.3631. To appear in Ann. Inst. Fourier (Grenoble).

[13] C. Faber and G. van der Geer, Sur la cohomologie des systèmes locaux sur les espaces des modules des courbes de genre 2 et des surfaces abéliennes, I, II. C.R. Acad. Sci. Paris, Sér. I, 338 (2004), 381-384, 467-470.

[14] C. Faber and R. Pandharipande (with an appendix by D. Zagier), Logarithmic series and Hodge integrals in the tautological ring, Michigan Math. J. 48 (2000), 215-252.

[15] C. Faber and R. Pandharipande, Hodge integrals, partition matrices, and the $\lambda_{g}$ conjecture, Ann. of Math. 157 (2003), 97-124.

[16] C. Faber and R. Pandharipande, Relative maps and tautological classes, J. Eur. Math. Soc. 7 (2005), 13-49.

[17] C. Faber and R. Pandharipande, Tautological and non-tautological cohomology of the moduli space of curves. Preprint, arXiv:1101.5489. To appear in the Handbook of Moduli.

[18] G. Faltings and C.-L. Chai, Degeneration of abelian varieties. With an appendix by David Mumford. Ergebnisse der Mathematik und ihrer Grenzgebiete (3), 22. SpringerVerlag, Berlin, 1990.

[19] W. Fulton, Intersection theory. Ergebnisse der Mathematik und ihrer Grenzgebiete (3), 2. Springer-Verlag, Berlin, 1984. 
[20] W. Fulton and J. Harris, Representation theory. A first course. Graduate Texts in Mathematics, 129. Readings in Mathematics. Springer-Verlag, New York, 1991. xvi+551 pp.

[21] E. Getzler, Intersection theory on $\bar{M}_{1,4}$ and elliptic Gromov-Witten invariants. J. Amer. Math. Soc. 10 (1997), no. 4, 973-998.

[22] E. Getzler, Resolving mixed Hodge modules on configuration spaces. Duke Math. J. 96 (1999), no. 1, 175-203.

[23] E. Getzler, The semi-classical approximation for modular operads. Comm. Math. Phys. 194 (1998), no. 2, 481-492.

[24] E. Getzler and M. Kapranov, Modular operads. Compositio Math. 110 (1998), no. 1, $65-126$.

[25] E. Getzler and R. Pandharipande, Virasoro constraints and the Chern classes of the Hodge bundle. Nuclear Phys. B 530 (1998), no. 3, 701-714.

[26] A. Givental, Gromov-Witten invariants and quantization of quadratic Hamiltonians. Mosc. Math. J. 1 (2001), no. 4, 551-568, 645.

[27] I. Goulden, D. Jackson, and R. Vakil, A short proof of the $\lambda_{g}$-conjecture without Gromov-Witten theory: Hurwitz theory and the moduli of curves. J. Reine Angew. Math. 637 (2009), 175-191.

[28] T. Graber and R. Pandharipande, Constructions of nontautological classes on moduli spaces of curves. Michigan Math. J. 51 (2003), no. 1, 93-109.

[29] T. Graber and R. Vakil, Relative virtual localization and vanishing of tautological classes on moduli spaces of curves. Duke Math. J. 130 (2005), no. 1, 1-37.

[30] J.L. Harer, Stability of the homology of the mapping class groups of orientable surfaces. Ann. of Math. (2) 121 (1985), no. 2, 215-249.

[31] T. Ichikawa, Teichmüller modular forms of degree 3. Amer. J. Math. 117 (1995), no. 4, 1057-1061.

[32] T. Ichikawa, Theta constants and Teichmüller modular forms. J. Number Theory 61 (1996), no. 2, 409-419.

[33] T. Ichikawa, Generalized Tate curve and integral Teichmüller modular forms. Amer. J. Math. 122 (2000), no. 6, 1139-1174.

[34] E. Ionel, Relations in the tautological ring of $M_{g}$, Duke Math J. 129 (2005), 157-186.

[35] R. Kaufmann, Yu. I. Manin, D. Zagier, Higher Weil-Petersson volumes of moduli spaces of stable n-pointed curves. Comm. Math. Phys. 181 (1996), no. 3, 763-787.

[36] S. Keel, Intersection theory of moduli space of stable n-pointed curves of genus zero. Trans. Amer. Math. Soc. 330 (1992), no. 2, 545-574.

[37] F.F. Knudsen, The projectivity of the moduli space of stable curves. II. The stacks $M_{g, n}$. Math. Scand. 52 (1983), no. 2, 161-199.

[38] F.F. Knudsen, The projectivity of the moduli space of stable curves. III. The line bundles on $M_{g, n}$, and a proof of the projectivity of $\bar{M}_{g, n}$ in characteristic 0. Math. Scand. 52 (1983), no. 2, 200-212.

[39] M. Kontsevich, Intersection theory on the moduli space of curves and the matrix Airy function. Comm. Math. Phys. 147 (1992), no. 1, 1-23.

[40] C.-C. M. Liu, K. Liu, and J. Zhou, Mariño-Vafa formula and Hodge integral identities. J. Algebraic Geom. 15 (2006), no. 2, 379-398.

[41] K. Liu and H. Xu, A proof of the Faber intersection number conjecture. J. Differential Geom. 83 (2009), no. 2, 313-335.

[42] K. Liu and H. Xu, Descendent integrals and tautological rings of moduli spaces of curves. Preprint, arXiv:0912.0584.

[43] E. Looijenga, On the tautological ring of $M_{g}$. Invent. Math. 121 (1995), 411-419.

[44] I. Madsen and M. Weiss, The stable moduli space of Riemann surfaces: Mumford's conjecture. Ann. of Math. (2) 165 (2007), no. 3, 843-941.

[45] A. Marian, D. Oprea, and R. Pandharipande, The moduli space of stable quotients. Preprint, arXiv:0904.2992.

[46] M. Mirzakhani, Weil-Petersson volumes and intersection theory on the moduli space of curves. J. Amer. Math. Soc. 20 (2007), no. 1, 1-23.

[47] S. Morita, Generators for the tautological algebra of the moduli space of curves. Topology 42 (2003), 787-819. 
[48] D. Mumford, Towards an enumerative geometry of the moduli space of curves. In Arithmetic and Geometry (M. Artin and J. Tate, eds.), Part II, Birkhäuser, 1983, 271-328.

[49] A. Okounkov and R. Pandharipande, Gromov-Witten theory, Hurwitz numbers, and matrix models. In Algebraic geometry - Seattle 2005, Part 1, Proc. Sympos. Pure Math. 80, Amer. Math. Soc., Providence, RI, 2009, 325-414.

[50] R. Pandharipande, A geometric construction of Getzler's elliptic relation. Math. Ann. 313 (1999), no. 4, 715-729.

[51] R. Pandharipande, The kappa ring of the moduli of curves of compact type: I, II. Preprints, arXiv:0906.2657, arXiv:0906.2658.

[52] R. Pandharipande, The kappa classes on the moduli spaces of curves. Preprint, arXiv:1108.5984.

[53] R. Pandharipande and A. Pixton, Relations in the tautological ring. Preprint, arXiv:1101.2236.

[54] D. Petersen, The structure of the tautological ring in genus one. Preprint, arXiv:1205.1586.

[55] M. Tavakol, The tautological ring of $M_{1, n}^{c t}$. Preprint, arXiv:1007.3091.

[56] M. Tavakol, The tautological ring of the moduli space $M_{2, n}^{r t}$. Preprint, arXiv:1101.5242.

[57] O. Tommasi, Rational cohomology of $M_{3,2}$. Compos. Math. 143 (2007), no. 4, 9861002.

[58] E. Witten, Two-dimensional gravity and intersection theory on moduli space. Surveys in differential geometry (Cambridge, MA, 1990), 243-310, Lehigh Univ., Bethlehem, PA, 1991.

[59] S.A. Wolpert, Lectures and notes: Mirzakhani's volume recursion and approach for the Witten-Kontsevich theorem on moduli tautological intersection numbers. Preprint, arXiv:1108.0174. (This volume.)

[60] S. Yang, Calculating intersection numbers on moduli spaces of pointed curves. Preprint, arXiv:0808.1974.

[61] Q. Yin, On the tautological rings of $M_{g, 1}$ and its universal Jacobian. Preprint, arXiv:1206.3783. 